NOTA TÉCNICA

\title{
RESISTENCIA AL ACHAPARRAMIENTO DEL MAÍZ MEDIANTE INFESTACIONES DE Dalbulus maidis EN MAÍZ ${ }^{1}$
}

\author{
Héctor Hidalgo ${ }^{2}$, Dan Jeffers ${ }^{3}$, Guillermo Castañon ${ }^{4}$, Flavio Rodríguez ${ }^{5}$
}

\begin{abstract}
RESUMEN
Resistencia al achaparramiento del maíz mediante infestaciones de Dalbulus maidis en maíz. Para detectar fuentes de resistencia al AM, se utilizó una metodología que consiste en infestar plántulas de maíz en los primeros quince días de edad. Se colocaron cuatro chicharritas infectadas con el complejo del achaparramiento del maíz (AM) por plántula. Se detectaron diferencias altamente significativas entre las líneas de maíz evaluadas con base en la producción de grano ( $\mathrm{p}=0,0001)$. Las líneas que sobresalieron son: LT- 173 con $3,818 \mathrm{~kg} / \mathrm{ha}$, LT- 183 con $3,808 \mathrm{~kg} / \mathrm{ha}$, LT -184 con 3,626 $\mathrm{kg} / \mathrm{ha}$. En cuanto a la manifestación de síntomas, LT-183 y LT - 184 no presentaron síntomas del AM pero LT-173 sólo presentó clorósis en el margen de la hoja y proliferación de hojas en la mazorca. Cuando se presentan bandas cloróticas en la base de las hojas generalmente había entrenudos cortos ( $\mathrm{r}=$ 0,83 ) a su vez, estos dos síntomas son los que en forma más persistente se presentan en el síndrome del AM $(r=0,45)$. Se discuten otras variables que influyen en la presencia del AM.
\end{abstract}

\begin{abstract}
Resistance to coro stunt spiroplasm (CSS) due to infestations of Dalbulus maidis. A method consisting of infesting maize plants during their first fifteen days was used to detect CSS resistance sources. Four leafuoppers infested with the CSS complex were placed on each seedling. Highly significant differences were found among lines evaluated according to grain yield ( $\mathrm{p}=0,0001)$. The best lines were LT173 with $3,818 \mathrm{~kg} / \mathrm{ha}$, LT-183 with $3,808 \mathrm{~kg} / \mathrm{ha}$, and LT - 184 with $3,626 \mathrm{~kg} / \mathrm{ha}$. LT-183 and LT-184 did not show any CSS symptoms, and LT-173 had only chlorosis in leaf borders and phylodia [proliferation of leaves in the ear]. Two closelyrelated symptoms are leaf chlorosis and short intemode $(\mathrm{r}=$ 0.83), which also appear most frequently in the CSS syndrome $(r=0.45)$. Other variables affecting CSS are also discussed.
\end{abstract}

\section{INTRODUCCIÓN}

El complejo del achaparramiento del maíz es un problema que se presenta con una fuerte incidencia en los países de Centro América, Celado et al. (1989); Lenardon et al. (1993); Gordón et al. (1995). En México se sabe de su incidencia pero ésta no está medida, sin embargo es común observar plantas con síntomas típicos de la enfermedad, Rodríguez y Preciado (1988).

La forma de control que se tiene actualmente es evitando la infección y esto se logra modificando las fechas de siembra. Existe una relación entre la fecha de siembra y la incidencia del complejo del achaparramiento; siembras tardías presentan menor porcentaje de plantas infec- tadas, Gordon et al. (1995). El mejoramiento genético para la resistencia a enfermedades es el método más económico, aunque es de largo tiempo. Este debe de incluir la identificación de enfermedades importantes, monitoreo de poblaciones del patógeno, desarrollo de métodos eficientes de inoculación, identificación del germoplasma resistente e incorporación de esa resistencia al germoplasma comercial, integrándolo con otros métodos de control, Pataky; Patrick (1995). Se han hecho intentos de mejoramiento genético en maíz para la resistencia a enfermedades en el trópico húmedo de México, Rodríguez y Preciado (1988) aunque en muchos casos no se utilizaron pruebas de patogenicidad y solo se ha evaluado el material en zonas donde el clima es propicio para el desarrollo de enfermedades.

1 Presentado en la XLIII Reunión Anual del PCCMCA en Panamá. 1997.

2 Campo Experimental Cotaxla, Km 34.5, Carretera Córdoba- Veracruz, Veracruz, México. Apdo. 453. CP 453. 3 Centro Internacional de Mejoramiento de Maíz y Trigo (CIMMYT), El Batán, Texcoco, México. 
El objetivo de este trabajo fue el de detectar fuentes de resistencia al complejo del achaparramiento del maíz mediante infestaciones artificiales de Dalbulus maidis los cuales fueron infectados con la enfermedad. El germoplasma resistente será utilizado en un programa de mejoramiento genético para la resistencia al complejo del achaparramiento.

\section{MATERIALES Y MÉTODOS}

El experimento se estableció en las instalaciones del Campo Experimental Poza Rica en Veracruz perteneciente al Centro Internacional de Mejoramiento de Maíz y Trigo (CIMMYT) en el periodo de verano. La siembra fue el 27 de julio y se cosechó el 14 de noviembre de 1996. Se establecieron dos lotes con grupos diferentes de materiales en un diseño experimental de bloques al azar con dos repeticiones. El primer grupo constó de 24 materiales y el segundo de 29. La unidad experimental fue de un surco de $2,5 \mathrm{~m}$ de largo y la distancia de siembra fue de 80 por $20 \mathrm{~cm}$, para una densidad de siembra de 62.500 plantas por hectárea. Se sembraron dos semillas por golpe y se deshijó para dejar solo una planta por sitio. Para infectar las plantas se requirió de chicharritas (Dalbulus maidis) que fueron criadas en las instalaciones de CIMMYT con el fin de tener un gran número para colocarlas en plantas infectadas con el Spiroplasma kunkelii el achaparramiento del maíz (CSS) y maize bushy stunt (MBS). La primera infestación fue con chicharritas infectadas con CSS. Se colocaron cuatro insectos por planta cuando esta tenía 12 días de edad y la segunda infestación se realizó a los 14 días de edad de la planta colocando la misma cantidad de chicharritas infectadas con MBS. A los 63 días de sembrado el experimento se midieron las variables, las cuales fueron de dos aspectos: una de ellas fueron características agronómicas de la planta, con la finalidad de observar si había una modificación en el comportamiento de la planta. Las principales características en este aspecto fueron: 1 altura de planta y altura de la mazorca, 2. Número de plantas, 3. número de mazorcas por plantas, 4. producción de grano. Las variables propias del complejo del achaparramiento fueron: a. síntomas que presentaron, b. número de plantas con síntomas, c. número de plantas secas y d. acame del tallo y raíz. Los síntomas que se evaluaron fueron 1. Bandas c10róticas en la base de las hojas 2. Entrenudos cortos 3. Clorosis severa en las hojas nuevas 4. enrojecimiento de la punta de las hojas 5. Proliferación de hojas en la mazorca en desarrollo (filodia) 6. Proliferación de mazorcas ( más de dos mazorcas por planta) 7. Clorosis en el margen de las hojas. Los resultados se analizaron como bloques al azar con el paquete estadístico SAS.

\section{RESULTADOS Y DISCUSIÓN}

\section{Síntomas presentados}

El patrón de la sintomatología que mostró la población de maíz indica (Cuadro 1) la variación del material genético con que se trabajó. Sólo dos líneas: LT-183 (AC-8223-4-2-1-2) y LT-184 (AC-8223-4-1-1-1) presentaron resistencia y se caracterizan por ser del mismo grupo, estos materiales poseen un 93,7 \% de endogamia. En el campo, al compararlas visualmente con el resto de los materiales, se observaron completamente sanas. Las líneas restantes presentaron de uno a seis síntomas de los siete esperados. Esta sintomatología concuerda con la señalada por De León (1984). El síntoma de clorosis severa en las hojas nuevas estuvo ausente, a pesar de que el síntoma de bandas cloróticas en la base de la hoja se observó en 48 de los 53 materiales. Existe la certeza de que todos los materiales fueron infectados y que las chicharritas (D. maidis) estuvieron el tiempo suficiente alimentándose para transmitir la enfermedad, por lo que los materiales que no presentaron síntomas del achaparramiento, poseen tolerancia. Sin embargo este trabajo no puede explicar en donde se encuentra la fuente de resistencia. Tingey (1985) menciona que algunos mecanismos de defensa de las plantas reside en aspectos morfológicos de la epidermis, tales como tricomas y/o sustancias alelopáticas que afectan el desarrollo y comportamiento de las chicharritas.

\section{Frecuencia de síntomas}

Al contabilizar los síntomas (Figura 1) en las dos repeticiones se observa que el síntoma con mayor frecuencia fue el enrojecimiento en la punta de la hoja (EPH) con 93 frecuencias, le siguen los entrenudos cortos (EC) y bandas cloróticas en la base de las hojas $(\mathrm{BCBH})$ con 83 y 81 frecuencias respectivamente. Esto demuestra la preminencia de estos tres síntomas en el síndrome del achaparramiento. Le sigue la proliferación de mazorcas (PM) con 41 frecuencias. Clorosis en el margen de las hojas (CMH) y proliferación de hojas en mazorcas en desarrollo (PHMD) tuvieron menos frecuencias. Un síntoma que se esperaba que apareciera con frecuencia es Clorosis severa en las hojas nuevas (CSHN) por ser un síntoma típico, y tuvo cere frecuencia de aparición. Hay que resaltar que hubo materiales que presentaron todos los síntomas dando un cuadro típico del achaparramiento, como lo fueron LT- $179 \mathrm{y}$ POB25COHC63-1-1-1-1-88-2-3-1-1.

\section{Incidencia de la enfermedad y rendimiento de grano}

El porcentaje de infección promedio fue de 67,8 con un rango de cero infección para las líneas LT-183 y 
Cuadro 1. Sintomatología del achaparramiento en 53 líneas de maíz a los 63 días de infestación con Dalbulus maidis infectados con Spiroplasma kunkelii (CSS) y maize bushy stunt (MBS).

\begin{tabular}{|c|c|c|c|c|c|c|c|}
\hline Líneas & ВCBH & EC & CSHN & EPH & PHMD & PM & CMH \\
\hline LT-173 (POB23-C-20-MH-450-1-3-1-1-1) & + & + & - & - & - & + & + \\
\hline LT -183(AC-8223-4-2-1-2) & - & - & - & - & - & - & - \\
\hline LT -184 (AC-8223-4-1-1-1) & - & - & - & - & - & - & - \\
\hline LT - 170 (POB22TSR-S2-21-2\#-1-1-1) & + & + & - & + & + & + & - \\
\hline LT -168 (STDC2-HC-43-1-1-1-2-3-3) & + & + & - & + & + & + & - \\
\hline LT -177 (POB22TSR-S2-21-2\#-1-1-5\#) & + & + & - & + & + & + & + \\
\hline LT -167 (STDC2-HC-43-1-1-1-2-1) & - & - & - & + & + & + & - \\
\hline LT -162 (POB22 TSR-S2-21\#-2\#-1-1-2\#) & + & + & - & + & + & + & - \\
\hline LT -172(AC-8149-5-1-2-1) & + & + & - & + & - & + & + \\
\hline LT $-166(\mathrm{LC}-36-1-4)$ & + & + & - & + & - & + & + \\
\hline LT -163 (POB22TSR-S2-21-2\#-1-1-3) & + & + & - & + & - & + & - \\
\hline LT -171 (LC-36-HC-\#-HC2-\#) & + & + & - & + & + & + & + \\
\hline LT -159 (POB43-113-1) & + & + & - & + & + & + & - \\
\hline LT $-156($ AC-7929-38-3-2-1) & + & - & - & + & - & + & + \\
\hline LT -165 ( TTC-17-62-2) & + & + & - & + & + & + & - \\
\hline LT -176 (PABG-ST-477-2-1-1-HC-1) & + & + & - & + & - & + & + \\
\hline LT -157(POB20TSR-20-2-2-3-2-1) & + & + & - & + & - & + & + \\
\hline $78-21-1-1-1$ & + & + & - & + & - & - & - \\
\hline $78-26-1-3-3$ & + & + & - & + & - & - & - \\
\hline LT -181 (ST-549) & + & + & - & + & - & + & + \\
\hline $78-26-2-1-2$ & + & + & - & + & - & - & - \\
\hline LT -158 (POOL23TSR40-2-3-2) & + & + & - & + & + & + & - \\
\hline $78-11-3-1-2$ & + & + & - & + & - & - & - \\
\hline LT -174 (POB.INT.DENT.5-1-2-HC-1) & + & + & - & - & - & - & + \\
\hline $78-26-2-2-2$ & + & + & - & + & - & - & - \\
\hline LT -164 (AC-7421-46-2-1-2) & + & + & - & + & + & + & + \\
\hline LT -169 (POB32C4HC-20-3-2-2-1-1) & + & + & - & + & - & + & + \\
\hline $11771-2$ & + & + & - & + & - & - & - \\
\hline POB21C5HC128-1-3-3-74-10-1-4-3 & - & - & - & - & - & - & + \\
\hline LT 178 (AC-7522-24-1-1-1-1-1) & + & + & - & + & - & - & + \\
\hline $78-16-1-2-1$ & + & + & - & + & - & - & - \\
\hline POB25COHC63-1-1-1-1-88-5-1-4-2 & + & + & -. & + & + & + & - \\
\hline $78-23-2-2-1$ & + & + & - & + & - & - & - \\
\hline POB21C5HC128-1-3-3-74-10-2-2-2 & + & + & - & + & - & - & + \\
\hline $78-23-1-4-2$ & + & + & - & + & - & - & + \\
\hline $78-11-3-1-2$ & + & + & - & + & - & - & - \\
\hline $78-16-1-3-1$ & + & + & - & + & - & - & - \\
\hline POB25COHC-63-1-1-1-1-88-2-3-1-1 & + & + & - & + & + & + & - \\
\hline LT -160 (TTC-206-2-1-1) & + & + & - & - & + & + & + \\
\hline LT - 179 (POB24-MH-97) & + & + & - & + & - & + & - \\
\hline LT -182 (D-471) & + & + & - & + & + & + & + \\
\hline $1177 \mathrm{~J}-4$ & + & + & - & + & + & + & - \\
\hline LT -180 (POB21C5-HC-28-1-3-3) & + & + & - & + & + & + & + \\
\hline LE-37-17-78-11-2-2-1 & + & + & - & + & - & - & - \\
\hline LT -161 (STDC2HC-43-1-1-1-2-2) & + & + & - & + & - & + & - \\
\hline LE-37-17-78-23-2-3-2 & + & + & - & + & - & - & - \\
\hline LE-37-17-78-23-1-3-2 & + & + & - & + & - & - & - \\
\hline POB25COHC63-1-1-1-1-88-5-1-2-3 & - & + & - & + & - & - & - \\
\hline $1177 \mathrm{~J}-5$ & + & + & - & + & - & - & - \\
\hline LE-37-17-80-4-1-1-3 & + & + & - & + & - & - & - \\
\hline 1177J-6 & + & + & - & + & - & - & - \\
\hline $1177 \mathrm{~J}-3$ & + & + & - & + & - & - & - \\
\hline LT -175 (D-7501-387-\#-1-\#) & + & + & - & + & - & + & + \\
\hline
\end{tabular}

* $\mathrm{BCBH}=$ bandas cloróticas en la base de las hojas; $\mathrm{EC}=$ entrenudos cortos; $\mathrm{CSHN}=$ clorosis severa en hojas nuevas; $\mathrm{EPH}=$ enrojecimiento en punta de las hojas; $\mathrm{PHMD}=$ proliferación de hojas en mazorcas en desarrollo; $\mathrm{PM}=$ proliferación de mazorcas (más de dos por planta); $\mathrm{CMH}=$ clorosis en el margen de las hojas. 


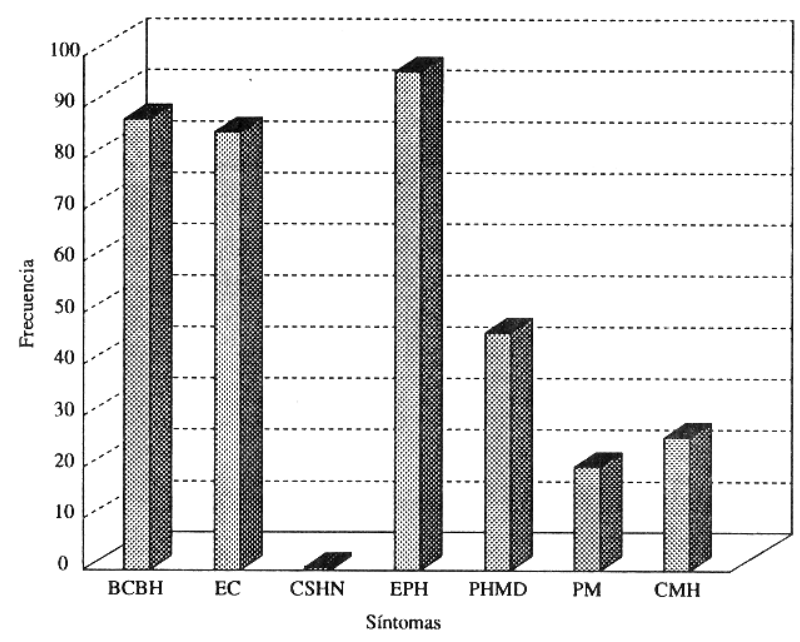

Figura 1. Síntomas en 53 líneas de maíz infestadas con Dalbulus maidis infectados con Spiroplasma kunkelli (CSS) y Maize bushy stunt (MBS).

LT-184, hasta $100 \%$ paraD-7501-387-I.\#-I-\#(Cuadro 2). Este porcentaje se midió con base en el número de plantas con uno o varios de los síntomas característicos. Se esperaba que conforme aumentaba el porcentaje de infección, en forma proporcional el rendimiento de grano disminuiría, sin embargo esto no fue así. Si se toma en cuenta que el rendimiento medio en este ensayo fue de $1.262 \mathrm{~kg} / \mathrm{ha}$, con un rango de $185 \mathrm{~kg} / \mathrm{ha}$ para LT-185 e incidencia de $92 \%$ de achaparramiento y LT-183 con $3.808 \mathrm{~kg} / \mathrm{ha}$ y $0 \%$ de infección. Uno de los materiales que presentó el mayor rendimiento fue LT-173 el cual, a pesar de arrojar un $35 \%$ de infección el rendimiento fue de $3.818 \mathrm{~kg} / \mathrm{ha}$. Otro caso fue el de POB43-113-1 que tuvo $56 \%$ de infección y el rendimiento fue de 2.878 $\mathrm{kg} / \mathrm{ha}$, que no es tan bajo como se podría esperar. Estos resultados manifiestan lo complejo que es la resistencia al achaparramiento si se toma en cuenta que el material con que se trabajó tiene alta endogamia ( 4 y ys). Los trabajos realizados por Celado et al. (1989) indican porcentajes de infección natural al achaparramiento del maíz de hasta $65 \%$ con rendimientos de $4.139 \mathrm{~kg} / \mathrm{ha}$ pero es con poblaciones SI y como se sabe, conforme aumenta el grado de endogamia, la producción de grano disminuye como sucedióen el presente trabajo.

\section{Correlaciones entre el maíz y achaparramiento}

El efecto de la enfermedad puede medirse con base en muchas variables y dependiendo del tipo de estas la correlación con respecto al rendimiento de grano puede ser negativa o positiva. Gordon et al. (1994) obtuvieron una correlación negativa entre la producción de grano y ,lantas con achaparramiento $(r=-0,55)$ así como producción de grano y mazorcas afectadas $(r=-$ 0,58 ). También señalan que el peso de la mazorca tuvo una correlación Ilegativa con el número de plantas $(\mathrm{r}=$ $-0,43)$ y número de mazorcas $(r=-0,44)$. Este tipo de correlaciones concuerdan con los resultados presentados en este trabajo si se toma en cuenta que cuando se mide el efecto de una enfermedad sobre un aspecto fundamental como lo es la producción de grano. De las correlaciones obtenidas en este trabajo (Cuadro 3) puede verse que fueron negativas entre la producción de grano y la presencia de bandas cloróticas en la base de la hoja $(r=-0,37)$; entrenudos cortos $(r=-0,34)$; enrojecimiento en la punta de la hoja $(\mathrm{r}=-0,36)$; el número de plantas secas $(r=-0,48)$; y número de mazorcas sin desarrollar $(\mathrm{r}=-0,38)$. Este resultado indica la importancia que tiene tomar en cuenta que los síntomas señalados tienen mayor peso en cuanto a la producción de grano se trata y que como resultado del avance de estos síntomas, se manifiesta la senescencia de las plantas afectando también el desarrollo de las mazorcas que en este caso, el crecimiento se detuvo cuando estaban en estado de jilote.

Tomando en cuenta otras correlaciones, se vio que la altura de la planta estuvo estrechamente relacionada con la altura de la mazorca $(\mathrm{r}=0,83)$ y producción de grano $(r=0,57)$. La altura de la planta y mazorca tienen una correlación positiva con la proliferación de la hojas en la mazorca ( $r=0,26$ y $r=0,27$ respectivamente). $\mathrm{La}$ correlación entre síntomas de la enfermedad pueden ser positivos, esto señala que se asocian entre sí y en algunos casos como las bandas cloróticas en la base de las hojas está estrechamente relacionada con la aparición de entrenudos cortos $(r=0,83)$ y en otros casos se contraponen, como lo es el caso en que cuando aparece el enrojecimiento en la punta de la hoja, se opone a la clorosis en el margen de las hojas $(r=-0,26)$ puede interpretarse entonces que cuando aparece primero uno de estos síntomas podr.ía inhibir al otro. El número de mazorcas sin desarrollo se correlacionó positivamente con las plantas secas $(r=0,40)$. Finalmente el acame del tallo se debe en parte a la aparición de bandas cloróticas en la base de las hojas. entrenudos cortos y el número de plantas secas $(r=0,23 ; r=0,28 ; r=0,33$ respectivamente) .

La infestación artificial con Dalbulus maidis y el complejo del achaparramiento es una técnica eficiente para seleccionar material de maíz resistente a CSS y MBS LT- 183 y LT- 184 son líneas de maíz con alta resistencia al complejo del achaparramiento del maíz.

Los síntomas: bandas cloróticas en la base de las hojas y entrenudos cortos están estrechamente relacio- 
Cuadro 2. Incidencia del achaparramiento del maíz medido en porcentaje de plantas infectadas y rendimiento de grano en kg/ha en 53 líneas de maíz.*

\begin{tabular}{|c|c|c|c|}
\hline Líneas & $\begin{array}{c}\text { Incidencia de } \\
\text { achaparramiento \% }\end{array}$ & $\begin{array}{r}\text { Rendir } \\
\text { kg/h }\end{array}$ & imiento \\
\hline LT - $183($ Ac-8223-4-2-1-2) & 0 & 3.808 & a \\
\hline LT - $184($ Ac-8223-4-1-1-1) & 0 & 3.626 & a \\
\hline $78-23-2-2-1$ & 33 & 863 & ghij \\
\hline LE-37-17-78-16-1-3-1 & 33 & 794 & ghij \\
\hline LT - 173 (POB23C-20-MH-450-1-3-1-1-1 & 35 & 3.818 & $\mathrm{a}$ \\
\hline LT-168 (STDC2-HC-43-1-1-1-2-3-3) & 44 & 2.274 & bc \\
\hline LE-37-17-78-16-1-2-1 & 44 & 15 & ghij \\
\hline POB21C5HC128-1-3-3-74-10-1-4-3 & 46 & 928 & fghij \\
\hline LT-167 (STDC2-HC-43-1-1-1-2-1 & 48 & 2.247 & bcd \\
\hline LT-162 (POB22TSR-S2-21\#-2\#-1-1-2\#) & 54 & 2.163 & bcde \\
\hline LE-37-17- 78-11-3-1-2 & 54 & 794 & ghij \\
\hline LE-37 17-78-26-1-3-3 & 55 & 1.184 & cdefghij \\
\hline LT-170 (POB22TSR-S2-21-2\#-1-1-1) & 56 & 2.878 & $a b$ \\
\hline POB21C5HC128-1-3-3-74-10-2-2-2 & 60 & 829 & ghij \\
\hline LT-174 (POB.INT.DENT.5-1-2-HC-1) & 61 & 1.073 & defghij \\
\hline LT-163 (POB22TSR-S2-21-2\#-1-1-3) & 61 & 1.934 & bcdefg \\
\hline LT-165 (TTC-17-62-2) & 61 & 1.507 & cdefghi \\
\hline LT-166 (LC-36-1-4) & 61 & 2.030 & bcdef \\
\hline LT-177 (POB22TSR-S2-21-2\#-1-15\#) & 62 & 2.258 & $\mathrm{bc}$ \\
\hline LT-174 (POB.INT.DENT.5-1-2-HC-1) & 66 & 1.074 & defghij \\
\hline LT-171 (LC-36-HC-\#-HC2-\#) & 68 & 1.816 & bcdefg \\
\hline LE-37-17-78-23-1-4-2 & 68 & 818 & ghij \\
\hline LE-37-17-78-26-2-1-2 & 70 & 1.137 & cdefghij \\
\hline LE-37-17-78-26-2-2-2 & 70 & 1.057 & efghij \\
\hline $11771-2$ & 71 & 928 & fghij \\
\hline LT-178 (Ac-7522-24-1-1-1-1-1) & 71 & 928 & fghij \\
\hline LE-37-17-78-21-1-1-1 & 73 & 1.243 & cdefghij \\
\hline LT-159 (POB43-113-1) & 73 & 1.809 & bcdefgh \\
\hline LT-157 (POB20TSR-20-2-2-3-2-1) & 73 & 1.346 & cdefghij \\
\hline $1177 \mathrm{~J}-6$ & 75 & 371 & $\mathrm{ij}$ \\
\hline LE-37-17-78-23-2-3-2 & 75 & 501 & $\mathrm{ij}$ \\
\hline LT-164 (AC-7421-46-2-1-2) & 77 & 980 & fghij \\
\hline $1177 \mathrm{~J}-3$ & 79 & 371 & ij \\
\hline LT- 158 (POOL23TSR40-2-3-2) & 79 & 1.113 & cdefghij \\
\hline LT-169 (POB32C4HC-26-3-2-2-1-1) & 79 & 928 & fghij \\
\hline РOB25СОHC63-1-1-1-1-88-5-1-2-3 & 80 & 464 & $\mathrm{ij}$ \\
\hline LT-180 (POB21C5-HC-28-1-3-3) & 80 & 621 & hij \\
\hline POB25COHC-63-1-1-1-1-88-2-3-1-1 & 83 & 754 & ghij \\
\hline LE-37-17-78-11-2-2-1 & 83 & 569 & $\mathrm{ij}$ \\
\hline LT-161 (STDC2HC-43-1-1-1-2-2 & 83 & 556 & $\mathrm{ij}$ \\
\hline LT-176 (PABG.ST-477-2-1-1-HC-1) & 84 & 1.427 & cdefghi \\
\hline POB25COHC63-1-1-1-1-88-5-1-4-2 & 85 & 899 & ghij \\
\hline LE-37-17-78-23-1-3-2 & 85 & 490 & $\mathrm{ij}$ \\
\hline LT-181 (ST-549) & 87 & 1.165 & cdefghij \\
\hline LT-172 (AC-8149-5-1-2-1) & 87 & 2.041 & bcdef \\
\hline РOB25COHC63-1-1-1-1-88-5-1-2-3 & 89 & 1.791 & bcdefgh \\
\hline $1177 \mathrm{~J}-5$ & 90 & 423 & $\mathrm{ij}$ \\
\hline LT-179 (POB24-MH-97) & 92 & 742 & ghij \\
\hline LT-175 (D-7501-387-\#-1-\#) & 92 & 185 & $\mathrm{ij}$ \\
\hline LT-182 (D-471) & 96 & 742 & ghij \\
\hline $1177 \mathrm{~J}-4$ & 100 & 620 & hij \\
\hline
\end{tabular}

* Las líneas de maíz fueron infestadas con Dalbulus maidis infectados con Spiroplama kunkelii (CSS) y Maize Bushy Stun (MBS).

**La variable producción de grano fue analizada con el paquete estadístico SAS. Rendimientos con la misma letra indica que so: iguales estadísticamente a un nivel de significancia de 0,01 . 
Cuadro 3. Características fenotípicas del maíz y su correlación con el achaparramiento a los 63 días de infestación con Dalbulus maidis; Spiroplasma kunkelii (CSS) y maize bbushy stunt (MBS).*

\begin{tabular}{|c|c|c|c|c|c|c|c|c|c|c|}
\hline & $\mathbf{A M}$ & PG & BCBH & EC & EPH & PHMD & PM & CMH & NPS & NMSD \\
\hline $\mathrm{AP}$ & 0,83 & 0,57 & & & & 0,26 & 0,43 & & & \\
\hline $\mathrm{P}<$ & 0,0001 & 0,0001 & & & & 0,007 & 0,0001 & & & \\
\hline $\mathrm{AM}$ & & 0,44 & & & & 0,27 & 0,38 & & & \\
\hline $\mathrm{P}<$ & & 0,0001 & & & & 0,004 & 0,0001 & & & \\
\hline PG & & & $-0,37$ & $-0,34$ & $-0,36$ & & & & $-0,48$ & $-0,38$ \\
\hline $\mathrm{P}<$ & & & 0,0004 & 0,0001 & 0,0001 & & & & 0,0001 & 0,0001 \\
\hline $\mathrm{BCBH}$ & & & & 0,83 & 0,26 & & & & 0,4 & 0,25 \\
\hline $\mathrm{P}<$ & & & & 0,0001 & 0,005 & & & & 0,0001 & 0,007 \\
\hline $\mathrm{EC}$ & & & & & 0,26 & & & & 0,45 & 0,29 \\
\hline $\mathrm{P}<$ & & & & & 0,005 & & & & 0,0001 & 0,002 \\
\hline EPH & & & & & & & & $-0,26$ & 0,32 & \\
\hline $\mathrm{P}<$ & & & & & & & & 0,007 & 0,0007 & \\
\hline PHMD & & & & & & & 0,32 & & & \\
\hline $\mathrm{P}<$ & & & & & & & 0,0001 & & & \\
\hline PM & & & 0,25 & 0,29 & & & & & & \\
\hline $\mathrm{P}<$ & & & 0,001 & 0,001 & & & & & & \\
\hline NMSD & & & 0,25 & 0,29 & & & & & 0,4 & \\
\hline $\mathrm{P}<$ & & & 0,007 & 0,002 & & & & & 0,0001 & \\
\hline AT & & & 0,23 & 0,28 & & & & & 0,33 & \\
\hline $\mathrm{P}<$ & & & 0,01 & 0,003 & & & & & 0,0004 & \\
\hline
\end{tabular}

* $\mathrm{AP}=$ Altura de la planta; $\mathrm{AM}=$ Altura de la mazorca; $\mathrm{PG}=$ Producción de grano; $\mathrm{BCBH}=$ Bandas cloróticas en la base de las hojas;EC= Entrenudos cortos; $\mathrm{EPH}=$ Enrojecimiento en la punta de la hoja; $\mathrm{PHMD}=$ Proliferación de hojas en mazorcas en desarrollo; $\mathrm{PM}=$ Proliferación de mazorcas (más de dos por planta); $\mathrm{CMH}=$ Clorósis en el margen de las hojas;NPS= Número de plantas secas; $\mathrm{NMSD}=$ Númerode mazorcas sin desarrollar.

nados y junto al enrojecimiento en la punta de las hojas, representan los síntomas característicos del achaparramiento del maíz.

\section{LITERATURA CITADA}

CELADO, M.R; MOREL,J.C.; CORDOVA-ORELLANA, H.S. 1989. Evaluación de líneas S1, resistentes al achaparramiento del maíz (Zea mays L.) provenientes de las poblaciones 28 y 36 del CIMMYT, ciclo 3. Programa Regional de Maíz de CIMMYT para Centro América y el Caribe, Panamá (Panamá). Proyectos colaborativos en agronomía, desarrollo y mejoramiento de germoplasma en maíz (Zea mays L.). Panamá (Panamá). IDIAP. p. 84-91.

DE LEON, C. 1984. Enfermedades del maíz. Una guía para su identificación en el campo. Centro Internacional de Mejoramiento de Maíz Trigo (CIMMYT). 3a. Edición.

GORDON, M.R.; De GRACIA, N.; FRANCO, J.; GONZALEZ, A. 1994. Evaluación de diferentes épocas de siembra y la relación con la incidencia del achaparramiento en el cultivo del maíz, Azuero, Panamá, 199394.

LENARDON, S.L.; LAGUNA, I.G.; GORDON, D.T.; TRUOL, G.; PANAMA, A.; GOMEZ, J.; BRADFUTE, O.E. 1993. Identification of corn stunt spiroplama in maize from Argentina. Plant Disease 77: (1) 100.

PATAKY, K.J.; PATRICK, RM. 1995. Successful use of resistance to control disease of corno Plant Disease 79: (12) $1256-1258$.

RODRIGUEZ MONTALVO, EA.; PRECIADO ORTIZ, RE 1988. Obtención de variedades resistentes o tolerantes a enfermedades foliares y de mazorca. Primera Reunión Científica y Agropecuaria. Centro de Investigaciones Forestales y Agropecuarias de Veracruz. INIFAPCIRGOC.MEX. p. 17.

TINGEY, WM. 1985. Plant defensive mecanisms against leafhoppers. In: Leafhoppers and planthoppers by L.R Nault and J.G. Rodríguez 1985. John Wiley and Sons. Inc. $217-233 \mathrm{p}$. 